\title{
RNAi technologies: a screen whose time has arrived
}

\author{
Researchers are finally reaping the rewards of large-scale RNA interference (RNAi) screens in \\ mammalian cells, obtaining insights into a wide range of biological questions. Nathan Blow takes a \\ look at the technology advances spearheading this revolution.
}

Large-scale RNAi screens in mammalian cells have moved into the research forefront in recent months. "There was a bit of lag time for people to get good at this, and to get through the first few rounds of screens," notes David Root, director of the RNAi Consortium at the Broad Institute in Cambridge, Massachusetts, USA; "it can be challenging, but it is working." A quick glance through PubMed reveals how broadly RNAi screening technology is now being applied-screens to profile cell division in cultured human cells ${ }^{1}$, to identify host factors important in HIV infection ${ }^{2}$, to find essential genes in human mammary cells ${ }^{3,4}$ and to locate the gene responsible for $5 q-$ syndrome $^{5}$ have all appeared in past 6 months.

With increasing numbers of short hairpin RNA (shRNA) and short interfering RNA ( siRNA) libraries available to researchers along with new systematic and pooled screening approaches, RNAi screens in mammalian cells are quickly becoming an option for more and more researchers. But as technology developers put all the pieces of the puzzle together, researchers are seeing that optimization and validation are still the most critical elements to obtaining meaningful data. "In general the more ambitious you get about what you want to learn from a cellbased screen," says Root, "the more challenging it is to get the right answer."

\section{On target with siRNA}

"There was a lot of skepticism when offtarget effects first came up, about whether RNAi screens would be useful," says Frank Buchholz of the Max Planck Institute of Molecular Cell Biology and Genetics in Dresden, Germany. The discovery that synthetic siRNAs could cause gene silencing in mammalian cells without the interferon responses previously observed when using double-stranded RNAs had set off a firestorm of interest. But the myth of RNAi as a 'silver bullet' for knocking down gene expression quickly evaporated when researchers began to see issues of off-target effects and variable potency.

A better understanding of off-target effects led to creative solutions, and recent advances in siRNA library design have focused on this lingering issue that wreaks havoc on screen results and complicates interpretation. Ambion of Austin, Texas, USA recently released a new version of a synthetic siRNA, called Silencer Select, which increases specificity through a combination of chemical modifications and better sequence-selection strategies to promote guide-strand uptake at low concentrations of siRNA. Other groups are taking a 'more is better' approach by pooling siRNAs in an effort to decrease individual siRNA concentration while maintaining potency, to minimize offtarget effects (see Box 1).

"Off-target effects with siRNAs are, in my view, completely addressable through appropriate experimental design," notes Christophe Echeverri, chief executive officer and chief scientific officer at Cenix BioSciences in Dresden, Germany. He thinks that although the ideal approach of rescue experiments remains technically challenging in most contexts, the more accessible solution, adopted by most researchers and companies today, is to test multiple siRNAs with different sequences against a target gene to increase the level of confidence in positive hits 6 . And he points out that that, in this regard, several suppliers now offer good large-scale genome-wide siRNA libraries.
Ambion and Applied Biosystems (Foster City, California, USA) have developed 19 siRNA libraries targeting the human and mouse genomes including sub-libraries targeting the druggable genome, kinases, phosphotases and G protein-coupled receptors. Dharmacon-Thermo Fisher in Lafayette, Colorado, USA, now offers over 51 different siRNA libraries targeting a wide variety of genes and pathways within the human genome, including membrane trafficking, insulin signaling,

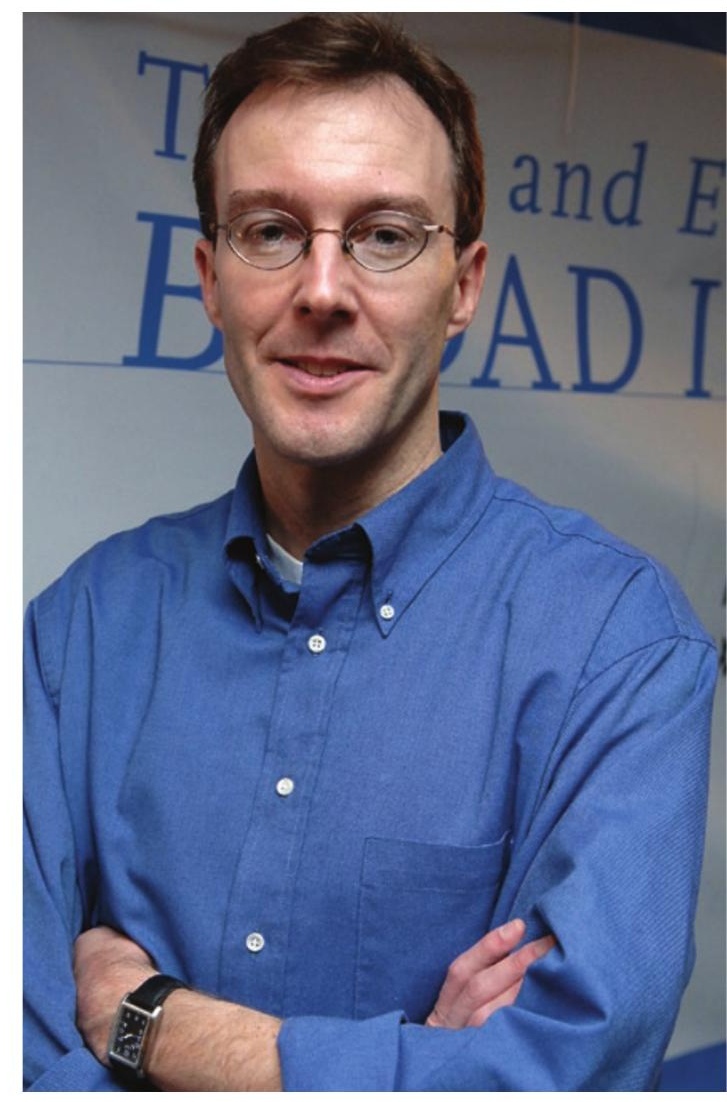

David Root and the RNAi consortium are working to develop new tools for genome-wide shRNA screens. 
cell-cycle regulation and ion channels. Qiagen, in Valencia, California, USA, also offers human, mouse and rat genomewide siRNA libraries in 96-well-plate formats. Echeverri notes that although each company relied on different algorithms to design siRNAs for their respective libraries, the statistics on silencing efficiency are very good and actually are quite similar.

The study of off-target effects of siRNAs has kept researchers and technology developers busy for years now, leading to both a deeper insight regarding the basic mechanism of RNAi and better siRNA tools. And Buchholz thinks that by having to go back to the drawing board, researchers now realize that the technology can deliver meaningful results when used in genetic screens.

\section{Short hairpin 2.0}

Beside siRNAs, there are shRNAs, longer stretches of RNA that are cleaved within cells into siRNAs to induce silencing. They are usually delivered by viral approaches, affording transduction in a wider range of cell types and longer silencing.

Stephen Elledge of Harvard Medical School in Boston, Massachusetts, USA, and Greg Hannon of Cold Spring Harbor

\section{BOX 1 TAKING LIFE ESI}

"esiRNA technology was developed at the same time RNAi hit mammalian cells," says Frank Buchholz. But unlike synthetic siRNA, getting endoribonucleaseprepared siRNA (esiRNA) technology into the hands of researchers is taking much more time.

When RNAi was first discovered in Caenorhabditis elegans and Drosophila melanogaster, it was found that long double-stranded RNA could induce gene silencing. Researchers then went on to find that these long double-stranded RNAs were actually processed and cleaved into siRNAs by an RNAse III enzyme called Dicer. "When this was found, we said, 'why don't we take the long double stranded RNA and do what is done in vivo in C. elegans in vitro?'," says Buchholz. Indeed, Dicer-digested double-stranded RNA induced a potent RNAi response in mammalian cells. Even better for Buchholz was their discovery that the processing also works with Escherichia coli RNAse III, which is much easier to make.

"One can play with the conditions such that the lengths of the fragments generated are the length necessary to perform RNAi experiments in mammalian cells," says Buchholz. Buchholz's group has now prepared an esiRNA for every gene in the human and mouse genome, which they are using to perform largescale RNAi screens.

The development of esiRNA has also provided greater insights into the use of siRNA as a research tool. "It has been fun to watch esiRNA evolve," says Christophe Echeverri; "the main thing we have learned is the value of highcomplexity pools." In recent years there has been much debate about the use of siRNA pools to target specific genes. So Buchholz and his colleagues decided to examine complex mixtures of both synthetic siRNA and esiRNA compared to single siRNAs. "We transfected cells with either chemically synthesized siRNA or esiRNA or a mixture of siRNA," he says, "and we saw that the more complex the mixture, the more you reduce off-target effects."

Buchholz thinks the reason for this is that within a complex mixture each siRNA is at low concentration, and the on-target effect of the multiple siRNAs is additive. Although each siRNA may also have an off-target effect, he notes that should be different for each siRNA in the mix and therefore the off-target effects will be diluted out.

Buchholz and others are hoping the esiRNA technology will soon be available to many more researchers interested in investigating the use of these siRNA pools. "We have had a lot of requests for the resource, but it has been difficult for us to meet the demand. It has yet to be commercialized, but soon we may have an option that would allow researchers to have access to ready-to-transfect esiRNAs." 
Laboratory in Cold Spring Harbor, New York, USA, have designed a second-generation version of their shRNA library. This second-generation version is available now through Open Biosystems of Huntsville, Alabama, USA, and according to Hannon contains more than 100,000 unique shRNAs targeting the entire human genome.

For this version of their library, Hannon and Elledge decided to express their shRNAs in the context of a microRNA (miR) cassette, which they refer to as shRNA-miRs. "The whole point of this second-generation library is that the vectors now work at single copy," notes Elledge, adding that single-copy integration is important for pooled screening applications.

But Elledge believes that improvements can be made even to the current secondgeneration library: "I think that the quality of the sequences that we have chosen could be improved as more information comes in about which hairpins work best and what the rules are." Hannon agrees and adds that as the quality of the sequences advances, and more individual clones have guaranteed potency and specificity, it will alleviate the need for having multiple shRNAs for each gene, and the number of clones in their library could decrease substantially.

\section{A consortium library}

"We quickly built up from nothing to a large-scale operation to create these libraries and put them into applications using high-throughput methods," says Root, describing the start of the RNAi Consortium at the Broad Institute. The goal of that first phase of the RNAi Consortium, which involved 11 different biomedical organizations, was to develop a lentiviral shRNA library to cover the human and the mouse genomes along with the capabilities to use that library in large-scale RNAi screens. Root says that by the end of this first phase, the RNAi Consortium's shRNA library contained around 180,000 clones-evenly divided between the human and the mouse genomes - targeting 17,000-18,000 genes for each.

The second phase of the RNAi Consortium started in April 2007 with several goals, including developing new strategies for arrayed screens and creating more specialized resources for screening and validation, such as vectors that allow integration of more than one shRNA in the same cell or that have different markers for use in fluorescence-assisted cell sorting experiments. But the primary goal of this second phase is very similar to that of Hannon and Elledge's goal-improving the library. "A huge part of this stage is making more shRNAs for each gene and then assaying their effectiveness," says Root.

He says that for all the available shRNA libraries, only a minority of clones knock down the intended target gene strongly, 
making the need to improve library potency a universal goal for developers. Root notes that for the RNAi Consortium's library about $40 \%$ of clones knock down expression of their target gene by $70 \%$ or better.

\section{Jumping into the pool}

Despite these improvements, not everybody is convinced by shRNAs yet. "We still have the philosophy: 'siRNA if you can; shRNA if you must'-but really only if you absolutely must," says Cenix BioSciences' Echeverri. And many users share this philosophy: although progress with shRNA libraries has rapidly advanced, working with and producing viruses in the lab is not a trivial matter. "I think the performance has improved with these libraries, but if you are going to go in that direction you still have to make a massive investment in know-how and infrastructure," notes Echeverri.

But several technology advances are springing up that might make using these libraries more accessible to researchers. Whereas most shRNA screens have been performed through a systematic screening approach, where each virus and the cell type of interest has to be placed into an individual well of a plate, less laborintensive pooled screening approaches are starting to emerge. "I think it will be a lot easier for people to do the whole genome using pooled screens," says Elledge; “you don't need all the extensive equipment and investment in infrastructure."

Methods to identify shRNAs of interest are essential when pooling viruses. For this reason, Elledge and Hannon have been incorporating two different barcoding formats within the viral vectors of their library. "One is a random-sequence 60 -mer that we inserted into each vector, and then we also can use a region of the hairpin as a barcode, which we call a halfhairpin barcode," says Elledge.

To deconvolute the pooled shRNAs, Elledge and Hannon have been relying on amplification followed by hybridization of the barcode regions to a barcode microarray chip. "We have used both the barcode and the half-hairpin in the past," says Elledge, "but our most recent publication used only the half-hairpins since we did not have all the sequences of the barcodes." He notes that they are also getting closer to a 60-mer barcode microarray chip, but thinks this chip is going to take some time to optimize because there is potential for cross-hybridization between the 100,000 randomly generated 60-mers. In November 2007, Agilent Technologies of Santa Clara, California, USA, announced Hannon and Elledge would standardize their barcode technology on Agilent Technologies' microarray platform. Hannon thinks that using pooled approaches and standardizing the barcode technology on microarrays will allow siRNA screening to more rapidly move into the hands of researchers.

Root and the RNAi Consortium have also been exploring pooled screening approaches. "The pooled screening strategy is ultimately a readout of the abundance of the shRNA in a pool of cells treated one way versus a pool of cells 


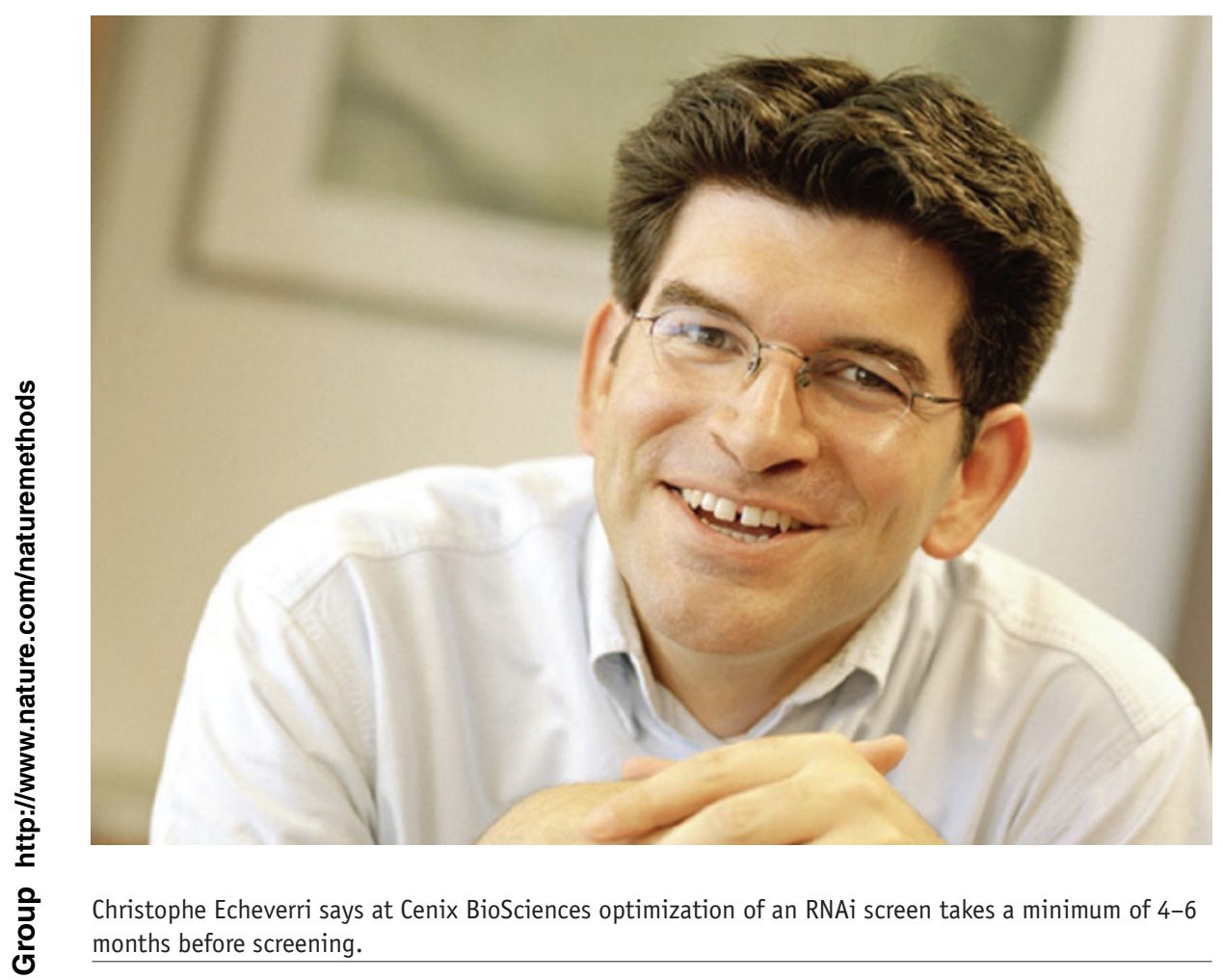

treated another," says Root, but he notes that some assays do not easily lend themselves to pooled approaches. In particular, many morphological phenotypes cannot be sorted using such an approach because researchers will not know which cells were treated with a specific shRNA when examining them after transduction.

Elledge thinks that if an assay does require a microscopic readout, researchers might be better served doing an siRNA screen opposed to using shRNA. But he does see the potential for technologies such as laser capture microdissection to be combined with pooled screens to isolate cells of interest, which could then be either cloned in a well or the insert could be amplified to determine the shRNA responsible for the observed phenotype.

\section{The new meaning of 'viral delivery'}

While Elledge, Hannon and the RNAi Consortium work out pooled approaches to shRNA screening, some companies are trying to make it easier for researchers to perform well-by-well systematic screens with shRNAs. "Traditionally, whenever you use lentiviral shRNA you have to produce the viral particles for each individual construct, which can be technically challenging, time-consuming and fairly expensive," says Supriya Shivakumar, global commercial marketing manager, functional genomics at St. Louis, Missouri, USA-based Sigma Aldrich. This led Sigma to develop LentiExpress, a plate-based reverse-transduction method for shRNA screening in which each virus particle containing a single shRNA is pre-arrayed in an individual well. "The researcher takes the plate out of the freezer, brings to room temperature and adds their cells," says Edward Weinsten, manager of operations, research biotech at Sigma Aldrich, "and as the cells adhere, they become infected."

This reverse-transfection approach developed in David Sabatini's lab at the Whitehead Institute for Biomedical Research in Cambridge, Massachusetts, USA, is used by many companies with siRNA libraries, but providing viral particles in such a single-well, arrayed format is just now starting to come on the scene. Sigma, a member of the RNAi Consortium, will be supplying portions of the shRNA library developed at the Broad Institute in this format. The first offering is a set of 3,109 shRNA clones representing 673 kinase genes arrayed onto 41 96well plates to screen the kinome.

While acknowledging that assay development is often the most difficult and time-consuming part of any RNAi screen, Shivakumar 
thinks LentiExpress will still make the screening portion considerably faster. "We were trying to take at least that part of the hurdle out of the way by having the reagents in an extremely easy-to-use format," she says.

Although the technology is falling into place and researchers are now obtaining novel biological insights using RNAi screens, many developers still think there is more work to be done. "RNAi screening works," says Root, "and we can improve it to be cheaper and more efficient. I think as more people want to do it, the drive to make it better will continue."

1. Kittler, R. et al. Nat. Cell Biol. 9, 1401-1412 (2007).

2. Brass, A.L. et al. Science $319,921-926$ (2008).
3. Silva, J.M. et al. Science $319,617-620$ (2008).

4. Schlabach, M.R. et al. Science 319, 620-624 (2008).

5. Ebert, B.L. et al. Nature 451, 335-339 (2008).

6. Echeverri, C.J. et al. Nat. Methods 3, 777-779 (2006).

Nathan Blow is the technology editor for Nature and Nature Methods (n.blow@boston.nature.com). 
SUPPLIERS GUIDE: COMPANIES OFFERING RNAI PRODUCTS

\section{Company}

Amaxa Biosystems

Ambion

Applied Biosystems

Agilent

Asuragen

BD BioSciences

Biocat

BioChain

Cellectricon

Cenix Biosciences $\mathrm{GmbH}$

Clontech

CombiMatrix

CytRx

Eurogentec

Exiqon

Geneservice

GeneTools

gen0way

Imgenex

Integrated DNA Technologies

Invitrogen

InvivoGen

Lentigen

Mirus Bio

Molecular Devices

MWG Biotech

New England Biolabs

Novagen

OligoEngine

Open Biosystems

Orbigen

Perkin Elmer

Polyplus Transfection

Promega

Qiagen

Regulus Therapeutics

Roche Applied Science

Rosetta Genomics

Sigma Aldrich

Stratagene

SuperArray Bioscience Corporation

Sylentis

Targeting Systems

Tebu-Bio

Thermo Fisher/Dharmacon

Vectalys

\section{Web address}

http://www.amaxa.com

http://www.ambion.com

http://www.appliedbiosystems.com

http://www.agilent.com

http://www.asuragen.com

http://www.bdbiosciences.com

http://www.biocat.de

http://www.biochain.com

http://www.cellectricon.com

http://www.cenix-biosciences.com

http://www.clotech.com

http://www.combimatrix.com

http://www.cytrx.com

http://www.eurogentec.com

http://www.exiqon.com

http://www.geneservice.co.uk

http://www.gene-tools.com

http://www.genoway.com

http://www.imgenex.com

http://www.idtdna.com

http://www.invitrogen.com

http://www.invivogen.com

http://www.lentigen.com

http://www.genetransfer.com

http://www.moleculardevices.com

http://www.mwg-biotech.com

http://www.neb.com

http://www.emdbiosciences.com

http://www.oligoengine.com

http://www.openbiosystems.com

http://www.orbigen.com

http://www.perkinelmer.com

http://www.polyplus-transfection.com

http://www.promega.com

http://www.qiagen.com

http://www.regulusrx.com

http://www.roche-applied-science.com

http://www.rosettagenomics.com

http://www.sigmaaldrich.com

http://www.stratagene.com

http://www.superarray.com

http://www.sylentis.com

http://www.targetingsystems.com

http://www.tebu-bio.com

http://www.dharmacon.com

http://www.vectalys.com 